\title{
Simulation Analysis of Fuel Mixture Influence on the Effects of Operation of Selected Steam Boilers in Municipal Districts
}

\author{
Dorota Wójcicka-Migasiuk' ${ }^{1}$ Magdalena Paśnikowska-Łukaszuk ${ }^{1 *}$ \\ ${ }^{1}$ Fundamental Technology Faculty, Lublin University of Technology, ul. Nadbystrzycka 38, 20-618, Lublin, \\ Poland \\ * Corresponding author's e-mail: m.pasnikowska-lukaszuk@pollub.pl
}

\begin{abstract}
The paper describes a system of steam boilers with the main attention drawn to the selected aspects of their operations in municipal district heating. The presented fuel characteristics show the characteristic factors in the described processes. The analysis reflects the simulations of fuel mixtures and their results in relation to the energy conversion processes in environmental aspects. Some considerations underline the results that have influence on the decisions pertaining to mixture composition. The paper presents comparative graphs and results in absolute terms. The conclusions are formulated taking into account the environmental effects, in particular localization of system elements and addresses the possible solution options to improve fuel mixtures and develop system efficiency.
\end{abstract}

Keywords: steam boilers, renewable energy sources, energy conversion, fossil fuels

\section{INTRODUCTION}

The development of modern technologies is still a quite substantial reason to increase the electric energy production, also in its part that comes from renewable sources [Wójcicka-Migasiuk, Paśnikowska-Łukaszuk, 2018]. That is why, despite many energy saving solutions and procedures, the need to produce more and more energy is still a leading imperative in industry and municipal life [Wójcicka-Migasiuk, 2016]. This, in turn, accelerates the research on optimizing the conditions of fuel burning and its efficiency effects at the lowest possible environmental impact.

Many plants, such as heat and power stations, continue their search for possibly effective fuel mixtures. Municipal heat and power station plants are very dependent on infrastructure, town supply system, local economy, geographical localization or even social and historical circumstances. Cities and other large urbanized areas are usually supplied from several plants cooperating in more or less unified systems. It is related with the availability of building connections and with the economical aspects when it is more convenient to divide their responsibility for the supply. Providing high reliability induces a cost increase through the need to maintain emergency units (and systems) in case of damage or any other failure in energy supply. Moreover, particular infrastructure of plants does not always allow covering larger agglomeration of districts. Nowadays, municipal heat distribution networks usually fully cover whole urbanized areas but some newly build districts go for individual solutions such as gas mains or power grid with separate connections or individual renewable energy supply such as photovoltaic panels or solar collectors, which in turn introduces some positive diversification also in district heating supply demand. It is worth to mention that the responsibility for heat supply in Poland is jointly taken by administrative institutions and heat and power station plants and they tend to sustainably develop these systems together with municipal infrastructure and urbanization progress. This process is widely recorded by media and is visible in reality [Gawlik, Mokrzycki, 2017]. 


\section{The example of a steam boiler system and steam-water unit basis for the analysis}

The system of operation and control in the exemplary plant is based on the computer controlled automatics providing direct communication between controllers, microprocessor adjusters and measurement data concentrators. The system enables to control processes by means of sequential control. Owing to a graphical interface, it is possible to visualize the whole system structure by means of diagrams and cooperating folders. The composition (presented in Fig. 2.) incorporates the following sub-control: main diagram for heat and power plant - ECE, steam - water boiler diagram - OR1, diagrams for steam boilers EKM2, EKM3, EKM5, and control subsystems for turbines, water softening units, compressors, refrigeration. The system is divided into two stations: operational and supervisor. The operational station tasks are: cooperation with controllers, adjusters and data concentrators which are placed in local control rooms. The supervisor station is located in particular rooms for individual authorized users.

The OR50-N K1 boiler presented in Fig.2 diagram is the main steam - water boiler in the system generation of heat and power. The effectiveness of this production is maximized through cogeneration and supported from two steam boilers: EKM2 unit and EKM5 unit. The main boiler (OR50-N K1) has a nominal steam output $50 \mathrm{t} / \mathrm{h}$ and its minimal steam output is in the range $\geq 30 \mathrm{t} / \mathrm{h}$ with outlet steam pressure 6.7 $\mathrm{MPa}$ (67 bar visible on a barograph in the system), outlet temperature below $490.0 \pm 5^{\circ} \mathrm{C}$, at minimal steam output, the temperature is $420^{\circ} \mathrm{C}$, water supply: $105^{\circ} \mathrm{C}$ which in total classifies this boiler as modern one of high nominal efficiency efficient, of a wide range of output capacity and highly effective conversion of chemical energy from fuel. This provides high efficiency in the periods of operation. Fuel is fed evenly, air is proportionally supplied to particular grate zones and cleaning system provides surfaces in the required condition.

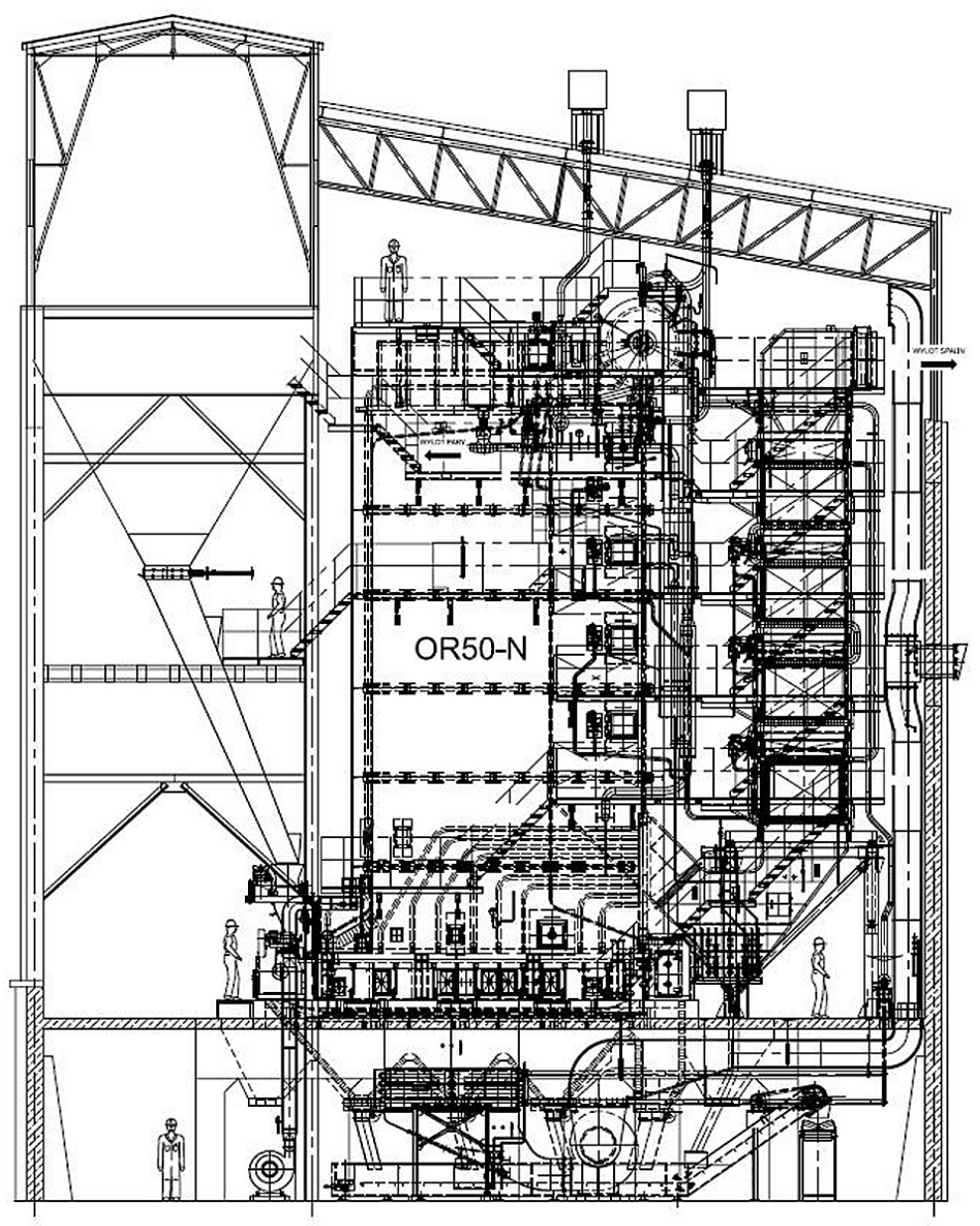

Fig.1. Boiler type OR in a plane diagram as a unit in the described system [Skrzypczyński, 2013] 


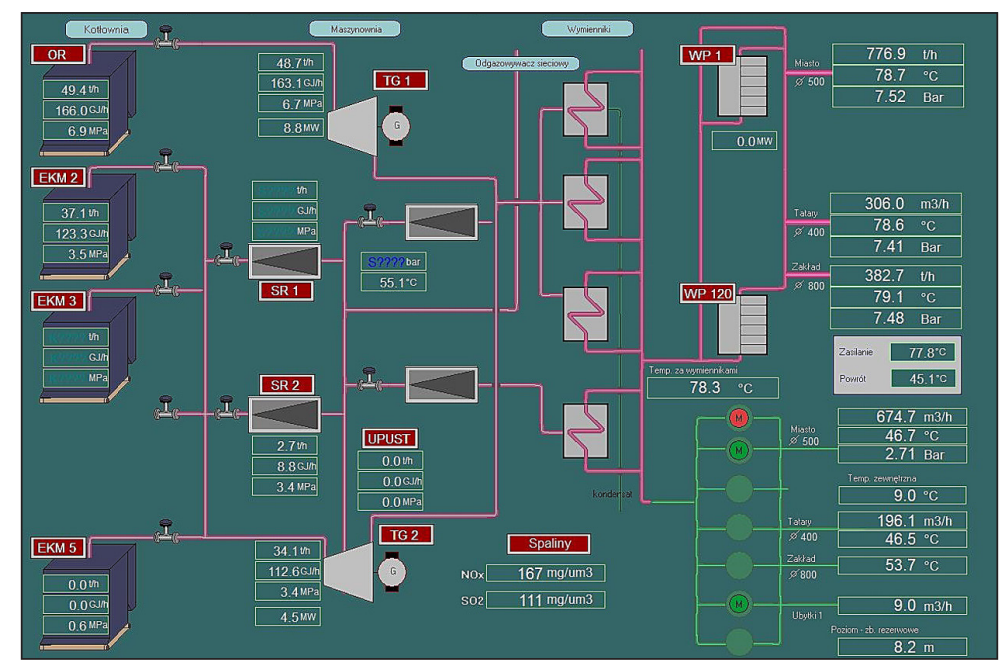

Fig. 2. Simplified diagram of system visualization including sub-systems of automatics

The control system incorporates complex automatic security, adjustment, alerts, measurements, executive units and through these enables to meet the required standards. This automatic system has so-called hierarchical decentralized structure. It incorporates the following main control elements: a separate case type AKAPiA for K1 boiler, RK1 - boiler distribution board, RNN - power (low voltage) distribution board. The measuring and executive units include: measurement sensors, converters and boiler drives. The control devices include: operational panel, drivers; drive inverters for: exhaust fume fans, preliminary air fan, grate drives, coal cart, and contactors. These devices are responsible for data collection and processing for the automatic technological process, verifying and correcting the measuring systems and executive units as well as sending control signals for the device drivers that are included in boiler systems.

The cooperation between the measuring system and executive units is necessary to convert physical quantities into standard signals identified by computer controlled devices. The measured quantities such as, for example: temperature, pressure, volume flow, mass capacity outflow are transmitted as current signal in the range between $4 \mathrm{~mA}$ and $20 \mathrm{~mA}$ to the boiler control board case (in this case - by means of electric wires). The awareness of all physical quantities and relevant signals is necessary to design parametric model analyses focused on continual modernization and adaptation of this heat and power station plant which is crucial to increase the efficiency of total energy production at minimal technological loss. These analyses have been continuously developed by the authors in parallel publications [WójcickaMigasiuk, Paśnikowska-Łukaszuk, 2018].

The electric systems in the control case incorporate relays, overload protection, differential protection for circuits of control phase, and direct measurement meters. They are important in continuous monitoring of water supply - flow rates, steam outlet temperature, injection water flow, steam boiler drum pressure and current water level in the steam boiler drum by an installed barograph. The incorporated automatic control system is activated in a local/basic mode, in which the circuits directly activating executive units are controlled (locally) or control units are controlled by a superior driver controller (basic). Control circuits consist of: control circuits for a coal cart, control circuits for fans of preliminary air, control circuits for a fume fan, control circuits for fans of secondary air and control circuits for valves.

The whole system provides appropriate performance of heat and power generation, supervised all day long not to disturb the process of burning fuels by any damage or accident with optional efficiency. Providing optional efficiency but at higher and higher levels is the purpose of the interdisciplinary research including the one described in this article.

\section{General characteristics of fossil fuels in aspects relevant to the presented considerations}

Fossil fuels, being constantly depleted, are still the most widely used source of energy. Coal, including lignite, crude oil, natural gas and peat, has been mined and extracted for ages, 
also opencast [Kosiarz, Maciejewski, 2016]. It is widely known that Poland has substantial coal resources which exert important economic pressure on this source of energy despite many risks to the environment and threats to the human health [Gawlik, Mokrzycki, 2017]. In such situation, the only right solution is to continue its use in cogeneration and centralized power plants supplying energy to municipal districts and industry with very high standards of dust and fume removal systems, with maximal energy retrofit and full recycling of waste. Assuming that global coal mining output is reduced in time focused on reduction of global basic emissions: $\mathrm{CO}_{2}, \mathrm{SO}_{2}, \mathrm{NO}_{\mathrm{x}}, \mathrm{CO}$ and dust emission. All landfill options must be put into practice, all so-called green production must be engaged and all possibilities to raise energy efficiency must be employed. The paper gives some contribution to this consideration.

The plant that is put as the example takes up the technology that uses a mixture of three resource outputs of domestic origin but at constant varying composition, depending on the current content of pollutants resultant from additives. Coal is rated as a sedimentary rock of plant origin and thus its natural additives vary quite significantly. That is why, the resulting fuel mixture is optimized in the aspects of maximized energy efficiency at reduced environment impacts, including emissions at the highest weight but not neglecting the others. Selected details are listed in Tables 1-3.

\section{Simulation analysis on fuel components and discussion of results.}

The use of fuel in the selected months is presented in Tables and Figures. The amounts were adjusted to ambient temperature, fuel content and its properties in particular periods. The combination of such mixtures provides best available results for efficiency and allows protecting the plant infrastructure against contamination with sulphur, dust and extensive emission from the plant. The ambient temperature determines the energy demand which is much higher in winter months than in the fall season. However, the fall season has been much warmer recently, its extension is observed and thus the total heating period is longer than it used to be. This has been explained by a global climate change [Braun, 2018]. Some reductions in total energy demand (especially electrical for lighting in buildings in eastern region of Poland) are expected if the so-called summer time is to be obligatory all year long.

It is visible from the graphs that October 2018 was Golden Polish Autumn which has not been regular recently, but last year occurred to be warm and sunny and thus the final energy production from the mixture could be reduced as seen in Table 10. November 2018 Table shows lower temperature values. The temperature in December was similar and thus the same three component mixture was used. Then January, had some warmer days which caused lower demand than in November. February 2019, despite winter season, was again warmer and additionally winter school holidays gave the effect of lower energy demand, especially from reduced hot water use in households were people left for vacation.

March was warmer and the energy demand lowered proportionally, which enabled heat and power production plants to reduce the maximal

Table 1. Component No. 1

\begin{tabular}{|c|c|c|c|c|}
\hline Characteristic parameter & Method & Unit & Result & $\begin{array}{c}\text { Allowable } \\
\text { deviation }(U)\end{array}$ \\
\hline Total water content $\left(\mathrm{W}_{t}^{\mathrm{r}}\right)$ & calculation & $\%$ & 13,5 & $\pm 1,2$ \\
\hline Moisture unbound content (Wex) & weighed & $\%$ & 8,9 & $\pm 0,9$ \\
\hline Analytical water content $\left(\mathrm{W}^{\mathrm{a}}\right)$ & weighed & $\%$ & 5,1 & $\pm 0,6$ \\
\hline Air-dry humidity - amf $\left(\mathrm{W}_{\mathrm{h}}\right)$ & weighed & $\%$ & 5,1 & $\pm 0,8$ \\
\hline Analytical ash content $\left(\mathrm{A}^{\mathrm{a}}\right)$ & weighed & $\%$ & 9,4 & $\pm 0,8$ \\
\hline Working state - ash content $\left(\mathrm{A}^{\mathrm{r}}\right)$ & weighed & $\%$ & 8,6 & $\pm 0,8$ \\
\hline Sulphur Total content $\left(\mathrm{S}_{t}{ }^{a}\right)$ & \multirow{2}{*}{$\begin{array}{l}\text { High temperature combustion } \\
\text { with IR detection }\end{array}$} & \multirow{2}{*}{$\%$} & 0,28 & $\pm 0,08$ \\
\hline Sulphur content $\left(\mathrm{S}_{\mathrm{t}}^{\mathrm{r}}\right)$ & & & 0,26 & $\pm 0,08$ \\
\hline Calorific value $\left(\mathrm{Q}_{\mathrm{s}}{ }^{\mathrm{a}}\right)$ & \multirow{3}{*}{ Calorific values computations } & \multirow{3}{*}{$\mathrm{kJ} / \mathrm{kg}$} & 27828 & \pm 323 \\
\hline Net calorific value $\left(Q_{s}{ }^{a}\right)$ & & & 26694 & \pm 325 \\
\hline Low calorific value $\left(Q_{s}{ }^{a}\right)$ & & & 24101 & \pm 442 \\
\hline Volatile component matter $\left(\mathrm{V}^{\mathrm{a}}\right)$ & \multirow{2}{*}{ weigh } & \multirow{2}{*}{$\%$} & 35,14 & $\pm 2,1$ \\
\hline Volatile matter ( $\left.\mathrm{V}^{\text {daf }}\right)$ & & & 41,10 & $\pm 2,1$ \\
\hline
\end{tabular}


Table 2. Component No. 2

\begin{tabular}{|c|c|c|c|c|}
\hline Characteristic parameter & Method & Unit & Result & $\begin{array}{c}\text { Allowable deviation } \\
(\mathrm{U})\end{array}$ \\
\hline Total water content $\left(\mathrm{W}_{\mathrm{t}}^{\mathrm{r}}\right)$ & calculation & $\%$ & 8,4 & $\pm 1,2$ \\
\hline $\begin{array}{l}\text { Moisture unbound content } \\
\left(\mathrm{W}^{\mathrm{ex}}\right)\end{array}$ & weighed & $\%$ & 5,5 & $\pm 0,9$ \\
\hline $\begin{array}{l}\text { Analytical water content } \\
\left(\mathrm{W}^{\mathrm{a}}\right)\end{array}$ & weighed & $\%$ & 2,8 & $\pm 0,6$ \\
\hline Air-dry humidity - amf $\left(\mathrm{W}_{\mathrm{h}}\right)$ & weighed & $\%$ & 3,1 & $\pm 0,8$ \\
\hline Analytical ash content $\left(\mathrm{A}^{\mathrm{a}}\right)$ & weighed & $\%$ & 15,6 & $\pm 0,8$ \\
\hline $\begin{array}{l}\text { Working state - ash } \\
\text { content }\left(A^{r}\right)\end{array}$ & weighed & $\%$ & 14,7 & $\pm 0,8$ \\
\hline Sulphur Total content $\left(\mathrm{S}_{\mathrm{t}}^{\mathrm{a}}\right)$ & \multirow{2}{*}{$\begin{array}{l}\text { High temperature combustion } \\
\text { with IR detection }\end{array}$} & \multirow{2}{*}{$\%$} & 1,10 & $\pm 0,08$ \\
\hline Sulphur content $\left(\mathrm{S}_{t}^{r}\right)$ & & & 1,04 & $\pm 0,08$ \\
\hline Calorific value $\left(Q_{s}{ }^{a}\right)$ & \multirow{3}{*}{ Calorific values computations } & \multirow{3}{*}{$\mathrm{kJ} / \mathrm{kg}$} & 27448 & \pm 323 \\
\hline Net calorific value $\left(\mathrm{Q}_{\mathrm{s}}{ }^{\mathrm{a}}\right)$ & & & 26471 & \pm 325 \\
\hline Low calorific value $\left(Q_{s}{ }^{a}\right)$ & & & 24745 & \pm 442 \\
\hline $\begin{array}{l}\text { Volatile component matter } \\
\left(V^{a}\right)\end{array}$ & \multirow{2}{*}{ weigh } & \multirow{2}{*}{$\%$} & 31,28 & $\pm 2,1$ \\
\hline Volatile matter $\left(\mathrm{V}^{\mathrm{daf}}\right)$ & & & 38,33 & $\pm 2,1$ \\
\hline
\end{tabular}

Table 3. Component No. 3

\begin{tabular}{|c|c|c|c|c|}
\hline Characteristic parameter & Method & Unit & Result & $\begin{array}{c}\text { Allowable deviation } \\
(\mathrm{U})\end{array}$ \\
\hline Total water content $\left(\mathrm{W}_{\mathrm{t}}^{\mathrm{r}}\right)$ & calculation & $\%$ & 10,3 & $\pm 1,2$ \\
\hline $\begin{array}{l}\text { Moisture unbound content } \\
\left(\mathrm{W}^{\mathrm{ex}}\right)\end{array}$ & weighed & $\%$ & 8,78 & $\pm 0,9$ \\
\hline $\begin{array}{l}\text { Analytical water content } \\
\left(W^{a}\right)\end{array}$ & weighed & $\%$ & 1,67 & $\pm 0,6$ \\
\hline Air-dry humidity - amf $\left(\mathrm{W}_{\mathrm{h}}\right)$ & weighed & $\%$ & - & $\pm 0,8$ \\
\hline Analytical ash content $\left(A^{a}\right)$ & weighed & $\%$ & 20,34 & $\pm 0,8$ \\
\hline $\begin{array}{l}\text { Working state - ash } \\
\text { content }\left(\mathrm{A}^{r}\right)\end{array}$ & weighed & $\%$ & 18,24 & $\pm 0,8$ \\
\hline Sulphur Total content $\left(\mathrm{S}_{\mathrm{t}}^{\mathrm{a}}\right)$ & \multirow{2}{*}{$\begin{array}{l}\text { High temperature combustion } \\
\text { with IR detection }\end{array}$} & \multirow{2}{*}{$\%$} & 0,49 & $\pm 0,08$ \\
\hline Sulphur content $\left(S_{t}^{r}\right)$ & & & 0,45 & $\pm 0,08$ \\
\hline Calorific value $\left(Q_{s}{ }^{a}\right)$ & \multirow{3}{*}{ Calorific values computations } & \multirow{3}{*}{$\mathrm{kJ} / \mathrm{kg}$} & 26359 & \pm 323 \\
\hline Net calorific value $\left(Q_{s}{ }^{a}\right)$ & & & 25393 & \pm 325 \\
\hline Low calorific value $\left(Q_{\mathrm{s}}{ }^{\mathrm{a}}\right)$ & & & 22950 & \pm 442 \\
\hline $\begin{array}{l}\text { Volatile component matter } \\
\left(\mathrm{V}^{\mathrm{a}}\right)\end{array}$ & \multirow[t]{2}{*}{ weigh } & \multirow[t]{2}{*}{$\%$} & 26,35 & $\pm 2,1$ \\
\hline Volatile matter ( $\left.\bigvee^{\text {daf }}\right)$ & & & 33,64 & $\pm 2,1$ \\
\hline
\end{tabular}

output availability by partial switching off maintaining the possibility to run maximal production again in case of weather failure. It can be seen that one component was excluded in that period and two components provided sufficient production.

\section{CONCLUSIONS}

The first general conclusions refer directly to the positive impact that has been obtained from a careful analysis of fuel mixture compositions produced on the basis of standardized fuel component test results. The obtained composition is optimal and enables the plant to keep reasonable resource reserve and thus maintain the required energy production availability. Then, the second conclusion refers to the concept of data collection by the authors who formulated the basis for further parametric analysis. Definitely, this set of data should be necessary to formulate models when consideration on physical processes and phenomena is not to be undertaken. The process of coal combustion will be analyzed from the point of the digital data introduced as numerical signals, where more input signals are used than outputs. The non-minimal phase models, as it was previously presented by the authors 
Table 4. Fuel consumption - October

\begin{tabular}{|c|c|c|c|}
\hline \multicolumn{4}{|c|}{ Fuel consumption [t] in boilers } \\
\hline Item & Component 1 & Component 2 & Component 3 \\
\hline 1. & 132,76 & 36,5 & 96,25 \\
\hline 2. & 109,85 & 48,23 & 94,76 \\
\hline 3. & 140,45 & 45,30 & 95,16 \\
\hline 4. & 97,71 & 115,18 & 117,11 \\
\hline 5. & 64,19 & 129,41 & 130,44 \\
\hline 6. & 27,87 & 90,96 & 126,17 \\
\hline 7. & 14,15 & 77,75 & 127,19 \\
\hline 8. & 47,29 & 38,26 & 118,23 \\
\hline 9. & 117,61 & 56,13 & 122,96 \\
\hline 10. & 114,6 & 50,14 & 128,91 \\
\hline 11. & 109,18 & 59,84 & 98,69 \\
\hline 12. & 70,62 & 14,79 & 111,66 \\
\hline 13. & 67,74 & 9,25 & 116,99 \\
\hline 14. & 75,04 & 13,16 & 123,75 \\
\hline 15. & 94,60 & 35,79 & 117,62 \\
\hline 16. & 90,12 & 31,15 & 117,94 \\
\hline 17. & 91,11 & 29,57 & 123,06 \\
\hline 18. & 89,48 & 24,62 & 129,71 \\
\hline 19. & 84,71 & 26,66 & 116,1 \\
\hline 20. & 95,13 & 39,24 & 111,78 \\
\hline 21. & 97,66 & 33,41 & 128,49 \\
\hline 22. & 92,87 & 114,74 & 120,23 \\
\hline 23. & 61,90 & 149,93 & 176,06 \\
\hline 24. & 0 & 129,62 & 259,24 \\
\hline 25. & 0 & 174,27 & 348,53 \\
\hline 26. & 0 & 108,14 & 0 \\
\hline 27. & 0 & 97,54 & 0 \\
\hline 28. & 0 & 115,74 & 0 \\
\hline 29. & 0 & 108,65 & 0 \\
\hline 30. & 0 & 86,84 & 0 \\
\hline 31. & 0 & 73,91 & 0 \\
\hline
\end{tabular}

Table 5. Fuel consumption - November

\begin{tabular}{|c|c|c|c|}
\hline \multicolumn{4}{|c|}{ Fuel consumption [t] in boilers } \\
\hline Item & Component 1 & Component 2 & Component 3 \\
\hline 1. & 0 & 71,51 & 143,02 \\
\hline 2. & 0 & 88,91 & 177,82 \\
\hline 3. & 0 & 79,15 & 158,3 \\
\hline 4. & 0 & 78,35 & 156,70 \\
\hline 5. & 0 & 79,26 & 158,51 \\
\hline 6. & 0 & 83,53 & 167,05 \\
\hline 7. & 0 & 98,41 & 172,32 \\
\hline 8. & 0 & 121,74 & 149,89 \\
\hline 9. & 0 & 136,57 & 151,45 \\
\hline 10. & 0 & 178,82 & 163 \\
\hline 11. & 0 & 209,90 & 209,9 \\
\hline 12. & 0 & 172,66 & 172,66 \\
\hline 13. & 0 & 144,29 & 144,29 \\
\hline 14. & 0 & 154,12 & 178,80 \\
\hline 15. & 0 & 202,24 & 202,24 \\
\hline
\end{tabular}

Table 5. cont.

\begin{tabular}{|c|c|c|c|}
\hline \multicolumn{4}{|c|}{ Fuel consumption [t] in boilers } \\
\hline Item & Component 1 & Component 2 & Component 3 \\
\hline 16. & 73,35 & 177,15 & 177,15 \\
\hline 17. & 344,88 & 68,55 & 137,11 \\
\hline 18. & 297,45 & 66,20 & 132,39 \\
\hline 19. & 288,94 & 66,13 & 132,26 \\
\hline 20. & 282,38 & 63,78 & 127,57 \\
\hline 21. & 297,55 & 52,69 & 135,50 \\
\hline 22. & 309,6 & 60,84 & 136,89 \\
\hline 23. & 225,8 & 96,60 & 191,19 \\
\hline 24. & 0 & 159,06 & 318,12 \\
\hline 25. & 0 & 147,64 & 295,28 \\
\hline 26. & 60,42 & 170,03 & 340,05 \\
\hline 27. & 124,06 & 169,81 & 339,62 \\
\hline 28. & 114,31 & 153,72 & 307,44 \\
\hline 29. & 201,45 & 161,30 & 322,59 \\
\hline 30. & 206,91 & 147,49 & 294,98 \\
\hline
\end{tabular}

Table 6. Fuel consumption - December

\begin{tabular}{|c|c|c|c|}
\hline \multicolumn{4}{|c|}{ Fuel consumption [t] in boilers } \\
\hline Item & Component 1 & Component 2 & Component 3 \\
\hline 1. & 0 & 71,51 & 143,02 \\
\hline 2. & 0 & 88,91 & 177,82 \\
\hline 3. & 0 & 79,15 & 158,3 \\
\hline 4. & 0 & 78,35 & 156,70 \\
\hline 5. & 0 & 79,26 & 158,51 \\
\hline 6. & 0 & 83,53 & 167,05 \\
\hline 7. & 0 & 98,41 & 172,32 \\
\hline 8. & 0 & 121,74 & 149,89 \\
\hline 9. & 0 & 136,57 & 151,45 \\
\hline 10. & 0 & 178,82 & 163 \\
\hline 11. & 0 & 209,90 & 209,9 \\
\hline 12. & 0 & 172,66 & 172,66 \\
\hline 13. & 0 & 144,29 & 144,29 \\
\hline 14. & 0 & 154,12 & 178,80 \\
\hline 15. & 0 & 202,24 & 202,24 \\
\hline 16. & 73,35 & 177,15 & 177,15 \\
\hline 17. & 344,88 & 68,55 & 137,11 \\
\hline 18. & 297,45 & 66,20 & 132,39 \\
\hline 19. & 288,94 & 66,13 & 132,26 \\
\hline 20. & 282,38 & 63,78 & 127,57 \\
\hline 21. & 297,55 & 52,69 & 135,50 \\
\hline 22. & 309,6 & 60,84 & 136,89 \\
\hline 23. & 225,8 & 96,60 & 191,19 \\
\hline 24. & 0 & 159,06 & 318,12 \\
\hline 25. & 0 & 147,64 & 295,28 \\
\hline 26. & 60,42 & 170,03 & 340,05 \\
\hline 27. & 124,06 & 169,81 & 339,62 \\
\hline 28. & 114,31 & 153,72 & 307,44 \\
\hline 29. & 201,45 & 161,30 & 322,59 \\
\hline 30. & 206,91 & 147,49 & 294,98 \\
\hline
\end{tabular}


Table 7. Fuel consumption - January

\begin{tabular}{|c|c|c|c|}
\hline \multicolumn{4}{|c|}{ Fuel consumption [t] in boilers } \\
\hline Item & Component 1 & Component 2 & Component 3 \\
\hline 1. & 0 & 161,50 & 323,01 \\
\hline 2. & 0 & 167 & 333,99 \\
\hline 3. & 73,9 & 164,62 & 403,13 \\
\hline 4. & 66,11 & 150,36 & 366,84 \\
\hline 5. & 49,87 & 160,46 & 375,6 \\
\hline 6. & 82,88 & 157,68 & 398,24 \\
\hline 7. & 108,92 & 162,80 & 434,51 \\
\hline 8. & 102,50 & 164,24 & 430,99 \\
\hline 9. & 86,49 & 128,08 & 417,84 \\
\hline 10. & 126,14 & 173,16 & 472,45 \\
\hline 11. & 81,35 & 108,98 & 299,32 \\
\hline 12. & 38,05 & 90,93 & 219,92 \\
\hline 13. & 0 & 177,34 & 354,69 \\
\hline 14. & 0 & 156,65 & 401,01 \\
\hline 15. & 46,44 & 171,40 & 389,25 \\
\hline 16. & 0 & 188,72 & 377,45 \\
\hline 17. & 0 & 170,70 & 341,41 \\
\hline 18. & 0 & 171,64 & 343,29 \\
\hline 19. & 57,48 & 163,31 & 392,48 \\
\hline 20. & 79,82 & 166,76 & 413,34 \\
\hline 21. & 172,33 & 50,73 & 416,45 \\
\hline 22. & 207,08 & 0 & 414,15 \\
\hline 23. & 111,78 & 109,25 & 442,06 \\
\hline 24. & 65,90 & 169,03 & 469,87 \\
\hline 25. & 66,19 & 148,95 & 430,29 \\
\hline 26. & 61,49 & 156,79 & 436,56 \\
\hline 27. & 30,42 & 155 & 370,84 \\
\hline 28. & 0 & 193,99 & 387,99 \\
\hline 29. & 0 & 196,93 & 393,87 \\
\hline 30. & 0 & 179,63 & 359,25 \\
\hline 31. & 0 & 169,84 & 339,68 \\
\hline
\end{tabular}

Table 8. Fuel consumption - February

\begin{tabular}{|c|c|c|c|}
\hline \multicolumn{4}{|c|}{ Fuel consumption [t] in boilers } \\
\hline Item & Component 1 & Component 2 & Component 3 \\
\hline 1. & 0 & 181,44 & 362,87 \\
\hline 2. & 0 & 161,09 & 322,17 \\
\hline 3. & 0 & 140,99 & 281,99 \\
\hline 4. & 0 & 162,9 & 325,80 \\
\hline 5. & 0 & 160,59 & 321,18 \\
\hline 6. & 0 & 154,93 & 309,86 \\
\hline 7. & 32,24 & 120,43 & 273,11 \\
\hline 8. & 0 & 167,72 & 335,45 \\
\hline 9. & 0 & 180,41 & 360,82 \\
\hline 10. & 0 & 164,26 & 328,52 \\
\hline 11. & 0 & 151,65 & 303,3 \\
\hline 12. & 0 & 156,99 & 313,98 \\
\hline 13. & 0 & 163,83 & 327,67 \\
\hline 14. & 0 & 161,33 & 322,67 \\
\hline 15. & 0 & 150,77 & 301,53 \\
\hline
\end{tabular}

Table 8. cont.

\begin{tabular}{|c|c|c|c|}
\hline \multicolumn{4}{|c|}{ Fuel consumption [t] in boilers } \\
\hline Item & Component 1 & Component 2 & Component 3 \\
\hline 16. & 0 & 167,24 & 334,49 \\
\hline 17. & 0 & 154,28 & 308,56 \\
\hline 18. & 0 & 137,83 & 275,66 \\
\hline 19. & 0 & 153,74 & 288,71 \\
\hline 20. & 0 & 148,80 & 297,59 \\
\hline 21. & 0 & 151,97 & 284,04 \\
\hline 22. & 31,66 & 167,04 & 356,17 \\
\hline 23. & 43,99 & 170,68 & 364,26 \\
\hline 24. & 8,99 & 159,93 & 311,06 \\
\hline 25. & 0 & 152,99 & 305,97 \\
\hline 26. & 0 & 140,8 & 281,61 \\
\hline 27. & 109,49 & 46,85 & 312,67 \\
\hline 28. & 139,66 & 0 & 279,32 \\
\hline
\end{tabular}

Table 9. Fuel consumption - March

\begin{tabular}{|c|c|c|c|}
\hline \multicolumn{4}{|c|}{ Fuel consumption [t] in boilers } \\
\hline Item & Component 1 & Component 2 & Component 3 \\
\hline 1. & 152,25 & 0 & 304,51 \\
\hline 2. & 235,91 & 0 & 420,77 \\
\hline 3. & 178,44 & 0 & 337,59 \\
\hline 4. & 123,33 & 0 & 246,65 \\
\hline 5. & 124,11 & 0 & 248,21 \\
\hline 6. & 152,56 & 0 & 305,13 \\
\hline 7. & 144,17 & 0 & 288,34 \\
\hline 8. & 129,09 & 0 & 258,17 \\
\hline 9. & 122,42 & 0 & 244,83 \\
\hline 10. & 139,38 & 0 & 278,75 \\
\hline 11. & 135,81 & 0 & 271,62 \\
\hline 12. & 148,57 & 0 & 297,14 \\
\hline 13. & 181,97 & 0 & 363,95 \\
\hline 14. & 152,81 & 0 & 305,63 \\
\hline 15. & 145,22 & 0 & 290,44 \\
\hline 16. & 154,85 & 0 & 309,70 \\
\hline 17. & 125,09 & 0 & 250,18 \\
\hline 18. & 126,97 & 0 & 253,93 \\
\hline 19. & 140,35 & 0 & 280,71 \\
\hline 20. & 145,65 & 0 & 291,29 \\
\hline 21. & 126,13 & 0 & 252,25 \\
\hline 22. & 109,76 & 0 & 219,51 \\
\hline 23. & 108,15 & 0 & 216,31 \\
\hline 24. & 106,56 & 0 & 213,11 \\
\hline 25. & 125,07 & 0 & 250,13 \\
\hline 26. & 163,1 & 0 & 326,2 \\
\hline 27. & 153,11 & 0 & 306,22 \\
\hline 28. & 139,85 & 0 & 279,7 \\
\hline 29. & 103,09 & 0 & 206,19 \\
\hline 30. & 82,92 & 0 & 165,83 \\
\hline 31. & 78,48 & 0 & 156,96 \\
\hline
\end{tabular}




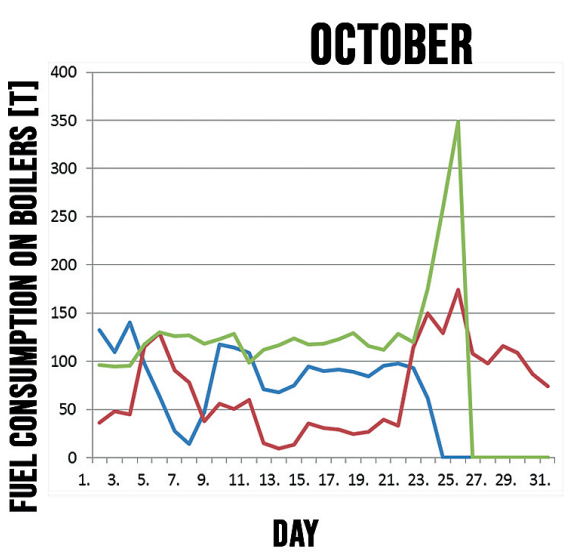

Fig. 3. Fuel consumption graph $[\mathrm{t}]$ divided into three components - October

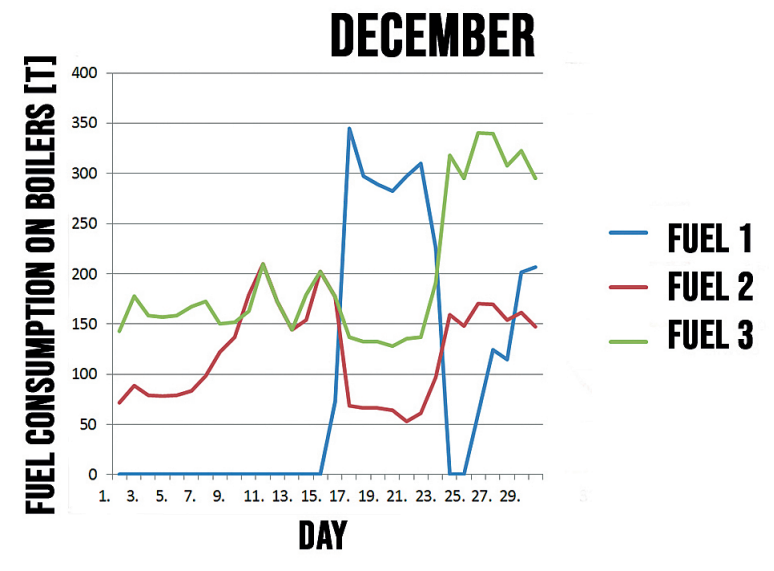

Fig. 5. Fuel consumption graph $[\mathrm{t}]$ divided into three components - December

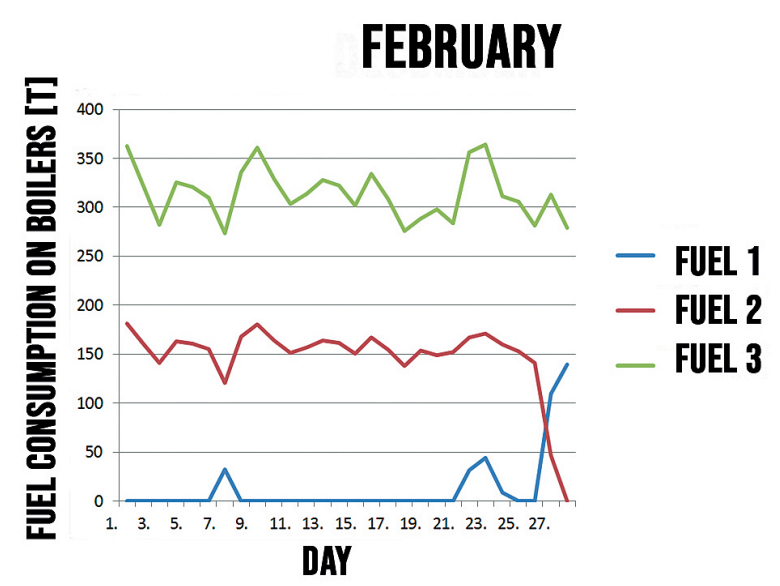

Fig. 7. Fuel consumption graph $[\mathrm{t}]$ divided into three components - February

[Wójcicka-Migasiuk, Paśnikowska-Łukaszuk, 2018], can help improve the controlling systems within the existing plant and also by thorough verifications could be transmissible onto numerous systems. Such mathematical attempt

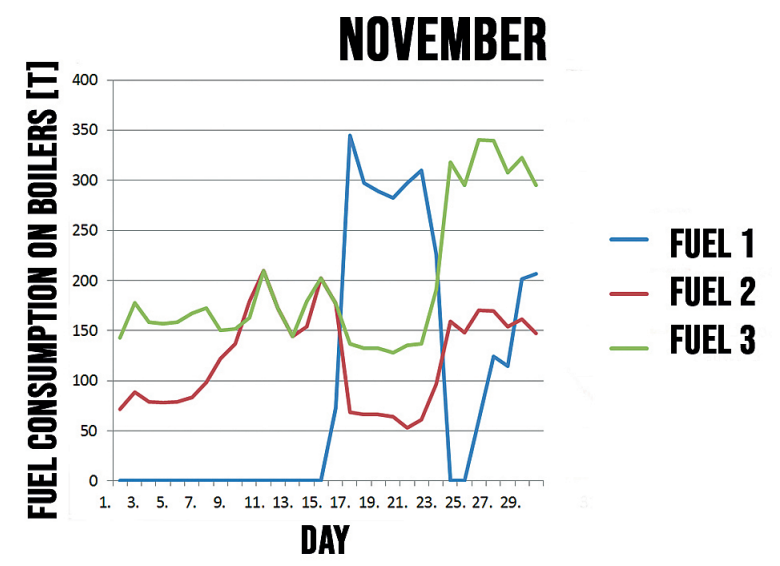

Fig. 4. Fuel consumption graph $[\mathrm{t}]$ divided into three components - November

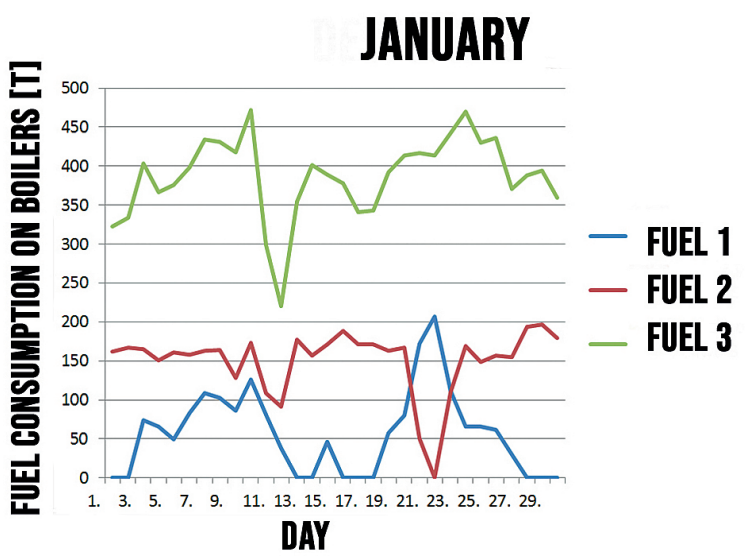

Fig. 6. Fuel consumption graph $[\mathrm{t}]$ divided into three components - January

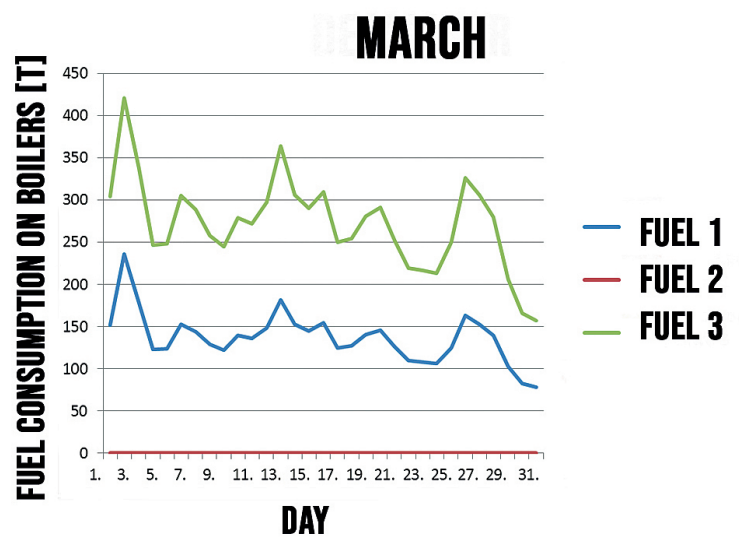

Fig. 8. Fuel consumption graph [t] divided into three components - March

has already been carried even into mathematical ecology [Waniewski,2018]; therefore, the authors would like to continue the pro-ecological attempts in traditional energy productions and preservation from harmful consequences. 
Table 10. Total energy output in October

\begin{tabular}{|c|c|c|c|c|c|c|}
\hline \multicolumn{3}{|c|}{ Component (net) calorific value kJ/kg } & \multicolumn{3}{|c|}{ Energy output [MJ] } & \multirow{2}{*}{$\begin{array}{l}\text { Total energy } \\
\text { output October }\end{array}$} \\
\hline Component 1 & Component 2 & Component 3 & Component 1 & Component 2 & Component 3 & \\
\hline 26694 & 26471 & 25393 & \multirow{2}{*}{53031368,16} & \multirow{2}{*}{57302303,12} & \multirow{2}{*}{85245062,79} & \multirow{2}{*}{195578734,1} \\
\hline \multicolumn{3}{|c|}{ Energy output - Total amount : } & & & & \\
\hline
\end{tabular}

Table 11. Total energy output in November

\begin{tabular}{|c|c|c|c|c|c|c|}
\hline \multicolumn{3}{|c|}{ Component (net) calorific value kJ/kg } & \multicolumn{3}{|c|}{ Energy output [MJ] } & \multirow{2}{*}{$\begin{array}{c}\text { Total energy } \\
\text { output } \\
\text { November }\end{array}$} \\
\hline Component 1 & Component 2 & Component 3 & Component 1 & Component 2 & Component 3 & \\
\hline 26694 & 26471 & 25393 & \multirow{2}{*}{75466607,4} & \multirow{2}{*}{96895771,95} & \multirow{2}{*}{172362379,4} & \multirow{2}{*}{344724758,7} \\
\hline Eners & output - Total a & unt : & & & & \\
\hline
\end{tabular}

Table 12. Total energy in January

\begin{tabular}{|c|c|c|c|c|c|c|}
\hline \multicolumn{3}{|c|}{ Component (net) calorific value kJ/kg } & \multicolumn{3}{|c|}{ Energy output [MJ] } & \multirow{2}{*}{$\begin{array}{l}\text { Total energy } \\
\text { output January }\end{array}$} \\
\hline Component 1 & Component 2 & Component 3 & Component 1 & Component 2 & Component 3 & \\
\hline 26694 & 26471 & 25393 & \multirow{2}{*}{45783947,16} & \multirow{2}{*}{119559712,7} & \multirow{2}{*}{165343659,9} & \multirow{2}{*}{330687319,8} \\
\hline \multicolumn{3}{|c|}{ output - Total amount : } & & & & \\
\hline
\end{tabular}

Table 13. Total energy in February

\begin{tabular}{|c|c|c|c|c|c|c|}
\hline \multicolumn{3}{|c|}{ Component (net) calorific value kJ/kg } & \multicolumn{3}{c|}{ Energy output [MJ] } & Total energy \\
output February
\end{tabular}

Table 14 Total energy in March

\begin{tabular}{|c|c|c|c|c|c|c|}
\hline \multicolumn{3}{|c|}{ Component (net) calorific value kJ/kg } & \multicolumn{3}{c|}{ Energy output [MJ] } & Total energy \\
output March
\end{tabular}

\section{REFERENCES}

1. Kosiarz E., Maciejewski M. 2016. Alternatywne metody wydobycia i wykorzystania węgla kamiennego. Kontrola i Audyt, Nr 2.

2. Gawlik L., Mokrzycki E. 2017. Paliwa kopalne w krajowej energetyce - problemy i wyzwania. Polityka Energetyczna - Energy Policy Journal, 20(4), 6-26.

3. Wójcicka-Migasiuk D., Paśnikowska-Łukaszuk M. 2018. Formulations of parametric models for energy and power system elements in the aspect of control quality improvments, Rynek Energii, 3(136).

4. Wójcicka-Migasiuk D., Paśnikowska-Łukaszuk M. 2018. Academic didactics and research aspects in solar energy education, Polska Energetyka Słoneczna, 1-4, 71-76.

5. Paśnikowska-Łukaszuk M., Wójcicka-Migasiuk D. 2017. Selected aspects of development towards energy efficient buildings. Journal of Ecological Engineering, 18(5), 137-143.
6. Urzędowski A., Wójcicka-Migasiuk D. 2016. Analiza zapotrzebowania, strat i zysków energii w budynku pasywnym z wykorzystaniem techniki termowizji, Tehnična Tvorčist-zbirnik naukovih prac.

7. Wójcicka-Migasiuk D., 2016. Wpływ zachowań proekologicznych na styl życia w warunkach polskich. Humanistyka a Nauki Ścisłe, 42-66.

8. Braun J., 2018. Bezpieczeństwo energetyczne jako dobro publiczne - miary i czynniki wpływające na jego poziom. Studia Ekonomiczne. Zeszyty Naukowe Uniwersytetu Ekonomicznego w Katowicach, Nr 358.

9. Waniewski J., 2018. Life Modeling Simulation (in Polish) - http://www.alife.pl/articles/p/waniewski2. html.

10. Skrzypczyński Z., Krokoszyński M. 2013. Nowoczesne parowe kotły rusztowe w elektrociepłowniach przemysłowych. Zakopane. Access: www.stc.pl/ dhttp.php?co=2013_04_09_softechnik.pdf 